\title{
Comunicación

\section{Políticas e industrias audiovisuales en México: apuntes y diagnóstico}

RODRIGO GÓMEZ GARCÍA ${ }^{1}$

Este artículo expone la pertinencia de estudiar a las industrias audiovisuales y a sus políticas públicas de forma integral. En primer lugar, se establece el procedimiento de construcción y el marco jurídico de las políticas públicas audiovisuales impulsadas en México durante los últimos veinte años. En segundo lugar, se presenta el estado actual de la industria audiovisual mexicana. En tercera instancia, se aportan indicadores industriales proporcionados por el Sistema de Clasificación Industrial de América del Norte (SCIAN) para observar la concentración audiovisual. Finalmente, se sugieren algunas líneas de trabajo para reformar las políticas audiovisuales con el propósito de impulsar y garantizar la diversidad cultural, la pluralidad política y la competencia económica.

PALABRAS CLAVE: políticas audiovisuales, industrias audiovisuales, concentración audiovisual, diversidad cultural.
This article addresses the relevance of the study of the audiovisual industries and their public policies in a comprehensive manner. First, it establishes the procedure for construction and legal framework of the audiovisual public policies and how are driven in Mexico over the past twenty years. Secondly, it presents the current state of Mexican audiovisual industry. In the third instance, offer industrial indicators provided by The North American Industry Classification System (NAICS) to monitoring audiovisual concentration. Finally, suggests some lines of work to reform the audiovisual public policies with the aim of promoting and safeguarding cultural diversity, political pluralism and economic competition.

KEY WORDS: audiovisual industries, audiovisual public policies, cultural diversity, audiovisual concentration.

1 Universidad Autónoma Metropolitana, campus Cuajimalpa.

Correo electrónico: rgomez@correo.cua.uam.mx,rgg28jaguar@gmail.com 


\section{INTRODUCCIÓN}

Este artículo parte del problema que plantea para una sociedad democrática el alto nivel de concentración económica de la industria audiovisual mexicana. Lo anterior afecta no sólo aspectos de orden económico en términos de competencia y desarrollo, sino también dimensiones de orden sociocultural (diversidad cultural) y políticas (pluralidad). Asimismo, se observa cada vez más una consolidación y una mayor penetración de los grupos empresariales de la industria audiovisual en la orientación, el diseño y la construcción de las políticas públicas relacionadas con el sector.

En primer lugar se presentan las coordenadas conceptuales para pensar a las industrias y a las políticas audiovisuales; posteriormente, se ofrece el marco jurídico en el cual estas se encuentran delimitadas y en tercer lugar, se describen los resultados arrojados por el Sistema de Clasificación Industrial de América del Norte (SCIAN). Estas cifras servirán para mostrar otro enfoque que permita observar la concentración económica de la industria audiovisual en México, al analizar de forma diferenciada sus distintos subsectores, ramas y subramas. Finalmente, se presenta una discusión, a partir de los insumos empíricos analizados, orientada a justificar los posibles cambios en las políticas audiovisuales para que incidan de forma directa en las industrias audiovisuales, y para establecer un balance entre las empresas privadas, el poder público y la sociedad.

\section{PUNTOS DE PARTIDA TEÓRICO-METODOLÓGICOS}

En este apartado presentaré los puntos de partida conceptuales, mismos que se orientan en dos direcciones: la primera establece cómo entiendo a las industrias audiovisuales mientras que la segunda plantea los conceptos y dimensiones claves para establecer el estudio de las políticas públicas audiovisuales.

Antes de entrar de lleno con las definiciones sobre las industrias audiovisuales, propongo recuperar cómo he definido a las industrias culturales (IC), pues justamente las audiovisuales se encuentran dentro de aquellas. Las IC conforman una parte de las instituciones que están directamente relacionadas con la producción social de significados, así 
como con la transmisión, renovación y apropiación del proceso de construcción de capital cultural y simbólico. Todo este proceso se encuentra administrado, producido, operado y distribuido bajo una lógica industrial capitalista, ${ }^{2}$ que tiene una gran penetración en la mayoría de nuestras sociedades, por lo que contribuyen con sus productos simbólicos en definir y distinguir la visión de nuestro mundo (Gómez, 2005:243-274). De ahí el interés por analizarlas y establecer su carácter cambiante, sus transformaciones económicas y la orientación de las políticas públicas que las delimitan (Mosco, 1996; Murdock, 1998:161-184).

En este momento, es importante precisar que mi acercamiento metodológico parte de una mirada histórico-estructural (Sánchez Ruiz, 1991; Golding y Murdock, 2000). Así, tanto las industrias audiovisuales como sus políticas públicas son abordadas desde una perspectiva histórica que observa a los diversos procesos económicos, políticos y socioculturales en constante transformación y en dinámicas de cambio, aunque al mismo tiempo con continuidades enraizadas a través del tiempo. ${ }^{3} \mathrm{De}$ tal forma que nuestras coordenadas de observación histórica observan tanto la continuidad como el cambio (Hesmondhalgh, 2002).

\section{Industrias audiovisuales}

Desde mi perspectiva debemos subrayar la necesidad de entender a las industrias audiovisuales en términos de operación económica e industrial, como cualquier otra industria aunque, como sabemos, al pertenecer al sector de las IC poseen distinciones específicas (simbólicas) que ninguna otra industria posee.

Para analizar a las industrias culturales propongo considerar cuatro elementos: 1) la lógica y la organización de sus empresas y mercados, así como su producción; ${ }^{4}$ 2) las innovaciones y aplicaciones tecnológi-

2 El término "industrias" está vinculado con los conceptos económicos de corte marxista: mercantilización, valor de cambio, concentración de capital y trabajo alienado.

3 Esto enmarcado dentro de la dinámica del capitalismo: naturaleza cíclica, crecimiento del capital monopólico, búsqueda de la maximización de las ganancias como objetivo principal, etcétera (Mosco, 1996).

4 Sin soslayar su desarrollo histórico y la conformación de sus mercados. 
cas -en este caso la convergencia-;5 3) los consumos y apropiaciones de sus bienes simbólicos, a través de los distintos canales de la comunicación social; y 4) la dinámica de construcción de marcos jurídicos y contextos democráticos. Es importante reparar que estas dimensiones no se observan de forma lineal, sino de manera diferenciada y a través de sus distintas interacciones, niveles y ámbitos.

La investigadora catalana Carmina Crusafon define a las industrias audiovisuales en los siguientes términos:

Por audiovisual entendemos aquella industria que pertenece al sector de la economía de la cultura, que produce bienes y servicios, que son el resultado de un conjunto de actividades que intervienen en la producción, distribución y exhibición de imágenes sobre distintos soportes. Se trata de una industria que tiene tres sectores principales: el cine, la televisión y el video, aunque los avances tecnológicos hacen que las fronteras entre ellos sean cada vez más borrosos y se integren nuevos servicios que se encuentran a caballo entre los tres sectores tradicionales. Asimismo, se caracteriza por tener una naturaleza dual, económica y cultural, que hace que sus actividades se encuentren en el centro de la encrucijada entre los siguientes ámbitos: la economía, la política y la cultura (Crusafon, 2000: 12).

5 Se entiende por "convergencia mediática" al último estadio desarrollado por la tecnología mediática, donde se pueden ofrecer conjuntamente productos, servicios y procesos de comunicación. Por primera vez en la historia, la tecnología brinda la posibilidad de tener en una sola innovación o plataforma todos los productos de las industrias audiovisuales (televisión, cine, música y radio), además de internet y otros servicios de telecomunicaciones. Esto se ha logrado a partir de "la unión de las comunicaciones en audio, video y datos en una sola fuente, recibida en un solo dispositivo, entregado por una sola conexión" (Forman y Saint John, 2000: 50). Actualmente, la tecnología ha logrado enlazar tres posibilidades: rapidez de conexión, capacidad de almacenaje y máxima calidad de imagen y sonido. Además, ha conseguido interactividad y movilidad, lo que le ha permitido al usuario -televidente o internauta-poder recibir, consultar, almacenar e interactuar con productos $\mathrm{y} / \mathrm{o}$ servicios audiovisuales, informáticos y documentales desde la comodidad de su casa, oficina o en movimiento en cualquier lugar y momento. 
Esta definición engloba de forma importante las distintas implicaciones que involucran a las industrias culturales en términos económicos, tecnológicos, políticos y socioculturales. Sin embargo, desde mi perspectiva, debemos considerar, principalmente, otros dos aspectos. Primero, dentro del sector de las industrias culturales antes que en el sector de la economía de la cultura, para entenderlas inicialmente dentro de una lógica de producción y organización particular compartida y, después, con los distintos subsectores y ramas que integran el amplio y difuso sector de la economía de la cultura, ${ }^{6}$ a partir de sus características simbólicas e identitarias. Segundo, recalcar la idea de definir a las IC en plural, pues si bien su carácter audiovisual (tecnológico) y cultural (simbólico) es su principal singularidad, cada una de ellas posee particularidades en sus formatos o en la circulación de sus productos, lo que las diferencia una de otras; su esencia plural nos ayuda a entender la existencia de matices y peculiaridades entre ellas. Por ejemplo, la industria de la televisión se caracteriza por su distribución en flujo (continua), mientras que la del video y la musical se da por edición (Miège, 1989:273-289; Bustamante, 2003; Herscovici; 2005). También es importante reflexionar en sus diferentes espacios de recepción comunicativa-salas de cine, hogares, telefonía móvil, espacios públicos...-, en sus diversas formas de acceso -renta, compra, pago por evento, etcétera- (Garnham, 1990; Herscovici, 2005), y en los soportes materiales e inmateriales de sus productos.

El Observatorio Europeo del Audiovisual nos ofrece otra definición operativa, en términos económicos, al describir a la industria audiovisual en ramas, con la intención de proveer información estadística que ayude a entender los constantes cambios del mercado. Según el Observatorio, el sector audiovisual se divide en cuatro ramas principales: a) la cinematográfica; b) la televisiva; c) la del video en casa; y d) otras formas de producción audiovisual: producción comercial, educativa y corporativa (European Audiovisual Observatory, 2003: 10-11).

6 Debemos recordar que la economía de la cultura es muy amplia y abarca desde los parques de diversiones, pasando por las industrias culturales hasta el turismo cultural (museos, entradas a sitios arqueológicos, parques nacionales, entre otros). 
A estas cuatro ramas tradicionales es necesario añadir el entretenimiento multimedia, es decir, los videojuegos en sus diferentes plataformas y los distintos recursos audiovisuales que encontramos en la red (idem). En esta rama multimedia es donde están ocurriendo las innovaciones y convergencias más novedosas de las industrias en cuestión, sobre todo en el terreno de la producción cultural. Pensemos en los videojuegos o en el broadcast yourself de Youtube.

En este sentido, coincido con García Canclini cuando dice

que la dinámica sectorial de las industrias audiovisuales no puede valorarse aisladamente, sino que se tiene que ver como un complejo entretejido de servicios múltiples (1999: 10).

Por lo tanto, a partir de ambas definiciones proponemos establecer a las industrias audiovisuales desde la interacción de cuatro dimensiones:

1. La económica e industrial, observándolas a través de la división de cuatro grandes subsectores ${ }^{7}$ cuyas fronteras, actualmente, con la convergencia tecnológica, se hallan cada vez más interrelacionadas y difusas: el cine, la televisión, el video 8 y los productos multimedia. Lo anterior nos ayuda, por el momento, a diferenciar y a establecer sus mercados y lógicas de producción y circulación.

2. Desde la política, al enmarcarlas dentro de los límites que fijan las distintas legislaciones, a través de los gobiernos locales, nacionales y supranacionales, con la idea de cumplir objetivos relacionados con el desarrollo democrático, económico y social, así como con la

7 A diferencia de la propuesta del Observatorio Europeo del Audiovisual, no pensamos a las IC en ramas sino en subsectores pues estos, a su vez, poseen distintas ramas y subramas. Así, a partir de la metodología del Sistema de Clasificación Industrial de América del Norte (SCIAN o NAICS, por sus siglas en inglés) se puede presentar una mejor sistematización de las distintas unidades económicas del sector audiovisual.

8 Estos sectores, a su vez, tienen distintas ramas. En el caso del cine es más sencillo caracterizarlas, pues consta de tres: producción, distribución y exhibición. 
diversidad y la creación cultural, en términos de acceso y derecho a la cultura. 9

3. Con base en su dimensión sociocultural, las concebimos a través de sus productos simbólicos, mismos que son generadores de imaginarios, identidades e ideologías. Todos estos elementos deben ser entendidos desde sus consumos específicos, sobre todo a partir de los contextos socioeconómicos regionales, nacionales e internacionales, y sus audiencias, diferenciadas en términos de capital cultural, de clase social y sus mediaciones. Sin soslayar las condiciones laborales en las cuales se desarrolla la producción cultural.

4) La dimensión tecnológica se entiende como la capacidad de producir, distribuir y exhibir imágenes en movimiento con sonido en sus diferentes soportes y plataformas. Esta situación ocurre en la actualidad de ida y vuelta, es decir, ahora las audiencias pueden producir y distribuir sus contenidos, aunque los canales de difusión masiva tradicionales (cine, radio y televisión) siguen siendo básicamente unidireccionales y controlados por los grandes agentes económicos de las industrias audiovisuales.

\section{Politicas audiovisuales}

Las políticas audiovisuales se deben entender en un marco más amplio que pertenece a las políticas de comunicación, ${ }^{10}$ y estas, a su vez, a las políticas culturales. ${ }^{11}$ Defino las políticas públicas audiovisuales como el conjunto de leyes, reglamentos, programas, acuerdos, acciones

9 En este último punto seguimos la propuesta de Cuilemburg y McQuail, 2003.

10 En otra investigación he profundizado en una definición amplia sobre las políticas de comunicación (Gómez, 2007).

11 García Canclini define a las políticas culturales como el conjunto de acciones que realizan diversos agentes para orientar el desarrollo simbólico, satisfacer las necesidades culturales y obtener consenso o disenso sobre un tipo de orden social. Las políticas culturales deben ser formuladas por varios agentes para generar un marco normativo en el que concursen todos los agentes involucrados; sólo así se podrá avanzar en el desarrollo cultural (1987). 
u omisiones que delimitan, configuran, protegen e impulsan al sistema audiovisual desde el poder político.

El investigador catalán Joseph Gifreu conceptualiza a las políticas de comunicación como las

series de principios y de normas establecidas para orientar el comportamiento de los sistemas de comunicación. Su orientación es fundamental y es a largo plazo, aunque pueden tener consecuencias operativas de importancia a corto plazo. Se moldean en el contexto del concepto en general que de la comunicación tiene la sociedad. [Se plantean de la misma manera] como emanan las ideologías políticas: de las condiciones sociales y económicas del país... (1986: 106).

Como complemento de estas definiciones, al igual que el planteamiento sobre el estudio de las industrias audiovisuales, considero que las políticas audiovisuales deben estudiarse a través de sus dimensiones económicas (reglas de competencia, apoyo a la producción, financiamiento, generación de fuentes de empleo y el papel de la industria en la economía del país), políticas (contexto democrático, lógicas del diseño y construcción de las políticas, es decir, desde qué posición se diseñan, implementan, evalúan y con qué fines), socioculturales (acceso, usos, apropiaciones y protección de los bienes comunicativos al entenderlos como valores culturales, ideológicos e identitarios) y tecnológicas (innovación e infraestructura). Por otro lado, es importante considerar que las políticas de comunicación surgen en el momento en el cual existe una interacción y una negociación entre la búsqueda de los intereses nacionales de los estados y las operaciones comerciales e industriales de las empresas privadas audiovisuales -situación que se presentó a principios del siglo XX-(Cuilenburg y McQuail, 2003).

Este planteamiento da pie a que recordemos la preocupación, la discusión y la participación que se dio en el seno de la UNESCO durante los años setenta y ochenta 12 por impulsar el diseño de políticas nacionales

12 Para una revisión sobre el desarrollo del debate sobre las políticas nacionales de comunicación a nivel internacional durante esos años, se recomienda revisar Gifreu (1986) y Ramos N. (2007). 
de comunicación en los distintos países considerados periféricos, ${ }^{13}$ pues se buscaba que estas políticas contribuyeran a conseguir beneficios democráticos, económicos, culturales y en materia de desarrollo social (McBride, 1978; Gifreu, 1986; Murciano, 1992). ${ }^{14}$ Estas sugerencias y debates, en la UNESCO, partían de la experiencia histórica del impulso y los resultados que las políticas normativas obtuvieron en las democracias occidentales avanzadas, principalmente las europeas ${ }^{15}$ (Cuilenburg y McQuail, 2003), así como por el problema que planteaban las inequidades existentes en materia de información y comunicación entre los países centrales y periféricosi6 (McBride, 1987).

En la actualidad, tanto las políticas de comunicación como las audiovisuales están siendo planteadas y diseñadas desde dos posiciones dominantes. La primera la ubicamos con la tradición económica liberal identificada con la ideología del libre mercado - esta postura tiende a la

13 En ese entonces se identificaban con el nombre de "países no alineados"; esto en el contexto bipolar de la Guerra Fría.

Sin embargo, en esas décadas la mayoría de los países periféricos no tenían regímenes democráticos consolidados, por lo que el impulso e implementación de dichas políticas nacionales de comunicación no tuvieron un respaldo democrático para llevar a buen puerto tanto sus objetivos y metas de corto como de mediano plazo. Además, en el terreno del debate internacional, los planteamientos suscitados por la UNESCO le trajeron serias críticas por parte de países como Estados Unidos y Gran Bretaña, por lo que dicha agenda de discusión dejó de ser una prioridad para la organización internacional y quedó relegada a un segundo término (Gifreu, 1986; Mattelart, 1998). Cabe subrayar que investigadores e intelectuales latinoamericanos tuvieron una participación muy activa y destacada sobre este particular.

15 Esas políticas nacionales estuvieron sustentadas en el contexto del nombrado paradigma de las políticas de comunicación de servicio público, el cual se desarrolló en varios momentos entre 1945 a 1980 y en la década de los noventa, teniendo como su principal objetivo el desarrollo democrático de sus sociedades (Cuilenburg y McQuail, 2003: 181-183).

16 Aquí nos referimos a las discusiones que se dieron en el marco de la propuesta de un Nuevo Orden Mundial Internacional de la Comunicación (NOMIC) dentro de la UNESCO. 
liberalización territorial- $\mathrm{y}$, la segunda, desde la tradición normativa, afín a un pensamiento socialdemócrata de intervención del Estado con visión territorial, ya sea nacional o regional.

La posición que promueve el libre mercado, en su vertiente neoliberal, 17 busca la no-intervención por parte de los gobiernos en los mercados de las comunicaciones. Esta concepción considera al Estado y a las instancias gubernamentales sólo como árbitros y promotores de los mercados, procurando una competencia efectiva y una infraestructura adecuada. Como señala el investigador británico Nicholas Garnham, detrás de estos presupuestos político-ideológicos se encuentra la retórica del libre mercado (1998: 214). Según esta postura liberal, las empresas privadas, a través de las leyes del libre mercado y de la competencia -la oferta y la demanda-, serán las que garanticen la diversidad de las expresiones políticas y culturales, así como un debate abierto dentro de sus sociedades y, por supuesto, "tarifas económicas y competitivas" para los consumidores. Además, se guían por el supuesto de que se le ofrece al público lo que quiere y pide a partir de sus consumos y de su libertad de elección (Qualter, 1992; Croteu y Hoynes, 2001).

Desde esta perspectiva, los productos audiovisuales no se diferencian del resto de las mercancías, y cualquier intervención gubernamental que busque regular los servicios comunicativos, al margen de maniobra de las empresas y sus estructuras de capital, puede ser acusada de atentar contra la libertad de expresión, de prensa y de libre competencia económica.

En contraparte, la tradición normativa de servicio público tiene objetivos y valores fincados en el bienestar social y económico. Procura garantizar la libertad de expresión, la diversidad cultural, el acceso universal, la calidad de contenidos y la responsabilidad social a través de la legitima intervención y participación de los estados en los mercados comunicativos con propósitos sociales, culturales, educativos y económicos (Pasquali, 1994; Richeri, 1994; Curran y Seaton, 1997; McQuail, 1998; Frau-Meigs, 2002; Bustamante, 2003). Desde la mirada del libre mercado, este tipo de regulaciones normativas son calificadas de proteccionistas, paternalistas y/o estatistas (Picard, 2002).

17 Nos referimos al impulso de políticas nacionales e internacionales que plantean la dominación de los negocios en todos los ámbitos sociales. 
Las políticas normativas se han centrado, sobre todo a partir de la experiencia internacional, en los siguientes aspectos: a) acceso universal; b) intervención o supervisión para que la actuación de las industrias audiovisuales se sustente con base en sus funciones y responsabilidades socioculturales ligadas al interés público, la diversidad cultural, la libertad de prensa, el pluralismo, la educación y la construcción de ciudadanía18; c) cuotas de pantalla a favor de la producción nacional; d) otorgamiento y renovación de concesiones y permisos no sólo bajo la figura de licitación; e) intervención en las estructuras económicas y financieras; f) controles a la propiedad cruzada de medios y a la propiedad extranjera; g) topes a la publicidad; h) subvenciones y financiamiento al sistema audiovisual y, en especial, a la producción audiovisual independiente nacional; e i) la promoción e impulso de circuitos audiovisuales no comerciales, a través de medios públicos, comunitarios y ciudadanos (Pasquali, 1994; Golding, 1998; McQuail, 1998; Sierra, 2002; Garnham, 2005).

De acuerdo con otras investigaciones, se advierte la conformación de un cambio en el balance que compone los valores políticos, económicos y sociales que delinean la definición del interés público que las industrias audiovisuales deben cumplir (McQuail, 1998; 2003; Sierra, 2006), así como su calidad de servicio público, pues cada vez se disloca más la discusión hacia los resultados económicos, relacionados con la eficiencia y el crecimiento (Picard, 2002), los cuales no necesariamente cumplen objetivos relacionados con el interés público ni con el desarrollo económico.

En cambio, la postura neoliberal hace a un lado la cuestión relativa a la pertinencia de implantar políticas proteccionistas y de servicio público, basadas en la necesidad de garantizar la diversidad cultural, las identidades nacionales, preservar el espacio público, el fomento a la pluralidad democrática y la iniciativa de corregir las posibles distorsiones que genera "la libre competencia" y las distintas fallas en las cuales incurren

18 Es importante señalar que la posición liberal no está en contra de estos objetivos, incluso también los busca la diferencia es que, desde su perspectiva, estos objetivos se pueden conseguir por la simple autorregulación de los mercados y la libertad de expresión. Es decir, el Estado no tendría que intervenir para que se cumplan dichas metas. 
los mercados dentro de las economías nacionales (Frau-Meigs, 2002: 3; Garnham, 2005: 16). Al respecto, García Canclini advierte que

a medida que las industrias culturales transnacionales se apropian de los ámbitos estratégicos de la vida pública, la cultura se privatiza y sufre un proceso de des-responsabilización respecto del interés social y las desigualdades (1999: 53).

De hecho, la posición de libre mercado es la que hasta el momento se presenta como hegemónica en las instancias supranacionales. Los más intensos debates se están suscitando en el seno de las rondas de la Organización Mundial de Comercio (OMC), 19 instancia que introduce los productos audiovisuales dentro de una lógica de negociación mercantil. Hasta el momento, dichas negociaciones se encuentran empantanadas pero a favor del libre mercado (Frau-Meigs, 2002; Turrent, 2002; Pawells y Loisen, 2003). Esta situación es importante subrayarla porque México, al lado de Estados Unidos, es uno de los países que apoya la posición del libre flujo de los productos y servicios audiovisuales a la par de cualquier otra mercancía. 20

\section{MARCO JURÍDICO DE LA INDUSTRIA AUDIOVISUAL Y LÓGICAS DE SU CONSTRUCCIÓN EN MÉXICO}

Es importante aclarar que, en México, la idea de entender a las industrias audiovisuales como un sector en sí mismo, es una perspectiva que ya

19 Los productos audiovisuales se discuten en torno a las mesas que plantea el Acuerdo General sobre el Comercio de Servicios (AGCS) y en el Acuerdo sobre los Aspectos de los Derechos de Propiedad Intelectual (AADPI), mejor conocido como TRIPS, por sus siglas en inglés.

20 En los tratados de libre comercio y de cooperación económica que ha signado México se promueve equiparar los productos audiovisuales como cualquier otra mercancía. El ejemplo más significativo de esta situación es el Tratado de Libre Comercio de América del Norte. Para una revisión a profundidad sobre el particular se recomienda revisar Galperin, 1999, Casas Pérez, 2006 y Gómez, 2006. 
se ha impulsado desde el ámbito académico (Toussaint, 1998; Sánchez Ruiz, 2001; 2004; García Canclini, 2006). En las legislaciones y en las acciones impulsadas por los distintos gobiernos mexicanos no ha existido un planteamiento en ese sentido; al contrario, son vistas como industrias independientes. Esta situación se expresa claramente en las variadas dependencias gubernamentales que tienen a su cargo algún aspecto de las industrias audiovisuales en nuestro país, es decir, se encuentran repartidas en varias secretarías e instituciones del Estado (por ejemplo, Secretaría de Comunicaciones y Transportes, Comisión Federal de Telecomunicaciones, Comisión Federal de Competencia, Secretaría de Gobernación, Secretaría de Educación Pública, Consejo Nacional para la Cultura y las Artes, etcétera). Esta dispersión de las industrias culturales a nivel de dependencias gubernamentales, también se observa en lo que se refiere a las distintas legislaciones y reglamentos. ${ }^{21}$ Cabe señalar que el Acuerdo de Convergencia (2006) y las recientes reformas a las leyes federales de Radio y Televisión y de Telecomunicaciones han comenzado a caminar en ese sentido de integración, pero no por sus características culturales, sino sólo en función de sus particularidades tecnológicas y de las oportunidades de negocio que ofrece la digitalización.

Otro punto que se debe subrayar es que tampoco han existido los mecanismos y los canales democráticos que permitan la participación de los distintos agentes sociales - al excluir a la sociedad civil-, para concursar en los procesos y servir de acompañamiento a la construcción de políticas públicas en materia audiovisual y de los sistemas comunicativos en general.22

21 Principalmente las leyes federales de Radio y Televisión con la de Telecomunicaciones; sin embargo, la de la industria cinematográfica sigue sin vincularse explícitamente a las dos mencionadas.

22 El más reciente y significativo ejemplo de esta situación fueron las mesas de diálogo a las cuales convocó en marzo del 2001 la Secretaría de Gobernación y el Senado de la República para diseñar la Reforma Integral de los Medios Electrónicos. Parte de los resultados de esas mesas de negociación fueron presentados en una reforma de ley a la Ley Federal de Radio y Televisión de 1960 en el Senado de la República. Sin embargo, esta propuesta de reforma nunca fue aprobada para su discusión en el pleno. En contrapar- 
Por otro lado, históricamente ha existido una clara falta de continuidad y de articulación en el impulso a las políticas de comunicación por parte de las distintas administraciones mexicanas desde 1934 hasta 1988 y, sobre todo, una ausencia de planeación integral entre los medios de comunicación y las telecomunicaciones. Asimismo una falta de visión de política de Estado en materia de comunicación. Como he documentado en otra investigación, las políticas fueron impulsándose sólo a partir de situaciones coyunturales23 (Gómez, 2007:389-430).

En contraparte, durante las administraciones de Carlos Salinas de Gortari, Ernesto Zedillo y Vicente Fox (1988-2006), las políticas audiovisuales fueron guiadas, principalmente, por sus especificidades económicas y tecnológicas con una clara convicción neoliberal. En ellas sólo se benefició a los grandes grupos empresariales del sector audiovisual (idem). Así, a partir de estas políticas de corte neoliberal se ha dejado el desarrollo, la innovación y la responsabilidad de las industrias culturales en manos de la iniciativa privada y bajo la lógica del libre mercado.

No obstante, cabe señalar que la problemática de las políticas públicas audiovisuales, al igual que en el resto de las políticas públicas en México, se entiende, en parte, en el contexto de un agotamiento del sistema político mexicano, caracterizado como "presidencialista, centralista, de uso oportunista de la ley, de partido único y de organizaciones corporativas dependientes" (Aguilar, 2004: 16). Lo anterior se refleja,

te, el presidente Vicente Fox decretó una serie de modificaciones que iban en sentido contrario a la iniciativa que había sido presentada en el Senado.

23 Recordemos la primera mitad del sexenio de Luis Echeverría cuando adquirió el Canal 13, iniciativa que obedeció a una estrategia de control directo de la información política de cara a la población, a raíz de la tensa situación político-social que el gobierno encaraba después de las represiones de 1968 y 1971. Es decir, la decisión de nacionalizar la televisora no buscaba incorporar o impulsar un sistema de televisión pública, sino que fue una respuesta estratégica frente a una situación particular que encaraba la administración de Echeverría. A partir de esa misma lógica, recordemos el decreto de Gustavo Díaz Ordaz, que obligaba a los concesionarios de la radio y la televisión comerciales a cederle al gobierno $12.5 \%$ de su tiempo de pantalla como pago de impuesto en especie (Gómez, 2006). 
todavía hasta nuestros días, en mayor o menor medida y según sea el sector o área de la vida pública, en el desprecio a las libertades políticas de los ciudadanos y la insuficiencia para asegurar la gobernabilidad del país (ibidem: 19).

De acuerdo con el politólogo Luis F. Aguilar, en el contexto de la transición a la democracia en México se observa que

la gobernabilidad democrática se basa tanto en un orden institucional apropiado, que hasta la fecha no se ha construido en nuestro país, y en un proceso decisorio articulado, robusto, con condiciones analíticas y operativas, que no puede ser alterado sin condenar la decisión al error práctico en sentido de ineficacia o ineficiencia (ibidem: 21 ).

En el caso de las políticas públicas audiovisuales, existen grandes rezagos en temas como la inclusión de la participación ciudadana en los procesos de construcción y decisión de dichas regulaciones. ${ }^{24}$ Las últimas reformas a las leyes federales de Radio y Televisión y de Telecomunicaciones - realizadas en diciembre de 2006- fueron uno de los ejemplos más abusivos y nítidos al respecto 25 (García Canclini, 2006; Gómez y Sosa, 2006). El anterior es sólo el caso más reciente en la tendencia histórica de las políticas públicas audiovisuales y de comunicación en México de no tomar en cuenta la participación y la opinión de otros actores de la sociedad civil. En el caso de la llamada Ley de Medios, se observó una variación dentro de esa continuidad: en esta ocasión existió conformidad, unanimidad y complicidad de todos los partidos políticos representados en la Cámara de Diputados, así como del poder Ejecutivo al publicar las reformas. Todo lo anterior se vio reflejado en el otorgamiento de más privilegios a las

24 Es importante recordar que la Constitución mexicana no permite a los grupos de la sociedad civil presentar o proponer iniciativas de ley; esto sólo pueden hacerlo las cámaras legislativas o el poder Ejecutivo.

25 Como sabemos, el abuso de la iniciativa fue tal que la Suprema Corte de Justicia de la Nación (SCJN), instancia conservadora por excelencia, declaró la inconstitucionalidad de tres artículos de la Ley Federal de Radio y Televisión y dos de la Ley Federal de Telecomunicaciones. 
empresas privadas de radiodifusión y de telecomunicaciones, así como una posición de subordinación de los partidos políticos ante ellas; todo esto dentro de un contexto de proceso electoral y a unas cuantas semanas de iniciar oficialmente las campañas electorales del 2006, hasta el momento los comicios más competidos en la historia del país y uno de los más polémicos.

Es importante anotar que las políticas audiovisuales en México se han cimentado desde 1988 al 2006 en cuatro pilares: 1) la privatización y el adelgazamiento paulatino de las empresas audiovisuales y de las telecomunicaciones en posesión del Estado - por ejemplo, Telmex, Imevisión, Cotsa y SATMEX-; 2) la rerregulación y la neorregulación de los mercados audiovisuales al priorizar las infraestructuras tecnológicas y los intereses de negocio de los grupos económicos dominantes -Ley de la Industria Cinematográfica (1992), Ley Televisa (2006), Ley Federal de Telecomunicaciones (1995 y 2006), Acuerdo por el que se adopta el Estándar Tecnológico de Televisión Digital Terrestre y se establece la Política para la Transición a la Televisión Digital Terrestre en México (2004), Acuerdo de Convergencia (2006), entre otros ordenamientos-; 3) la disminución de la intervención pública en la producción, distribución y exhibición de los productos audiovisuales (Galperín, 1999: 634), a través de subsidios y financiamiento; y 4) la apertura a la participación de capitales extranjeros en todos los rubros del audiovisual -por ejemplo, las cadenas de salas de cine Cinemark y Cinemex, la cadena Radiópolis y, en su momento, Televisión Azteca en su alianza con la NBC,26 etcétera- (Gómez, 2007).

Estas líneas de acción debemos entenderlas en un marco de reformas estructurales iniciadas por la administración de Miguel de la Madrid, con base en los lineamientos del llamado Consenso de Washington y aceleradas a través del Tratado de Libre Comercio de América del Norte (Crovi, 1997; Sánchez Ruiz, 2001; Gómez, 2006). Al mismo tiempo, dentro de un proceso de transición a la democracia en el cual aún imperan inercias de la cultura política heredara de un sistema político semiautoritario, construido a lo largo de setenta años, motivo por el cual la elite política no deja de expresar distintas prácticas antidemocráticas y corporativas.

26 Sobre el particular se recomienda revisar Gómez, 2004. 


\section{ESTADO ACTUAL DE LA INDUSTRIA AUDIOVISUAL} Y SU CARACTERIZACIÓN. DATOS SICAN 1999-2003

La estructura de las industrias audiovisuales, en México, la caracterizo como oligopólica, ya que sus mercados están dominados por un puñado de empresas (Sánchez Ruiz, 2003), así como altamente concentrada en las ramas relacionadas con la producción y la distribución televisiva, controladas casi en su totalidad por Televisa y TV Azteca. ${ }^{27}$ Asimismo, se observa que los capitales estadounidenses ya participan de forma ascendente en varias ramas de la industria, principalmente en las empresas de cable y de exhibición y distribución cinematográficas. Sólo en la televisión abierta son los capitales mexicanos los que predominan (Gómez, 2006). También se advierte que la balanza total de bienes audiovisuales (televisión, cine y video) está siendo dominada por los productos estadounidenses (Sánchez Ruiz, 2005; Ugalde, 2006), lo que confirma la tendencia observada a finales de los años noventa de ser una industria deficitaria en términos globales (Sánchez Ruiz, 2001).

Por razones de espacio, en este artículo presento solamente un análisis de los datos económicos e industriales que arroja el Sistema de Clasificación Industrial de América del Norte (SCIAN) con base en los censos económicos de 1999 y 2003, a partir de tres indicadores: a) unidades económicas; b) personal ocupado y c) producción bruta total. Estos datos buscan complementar los análisis realizados en materia de concentración y del estado de la cuestión de las industrias audiovisuales en México (Sánchez Ruiz, 2005; Gómez, 2006).

Un punto importante a destacar sobre los datos aquí analizados es que ofrecen elementos contradictorios generados por las políticas públicas impulsadas por las administraciones Salinas-Zedillo-Fox dentro de la misma lógica de sus presupuestos de libre mercado, sobre todo en términos de prácticas monopólicas y falta de generación de empleos.

27 Estas dos empresas se reparten 95\% de la inversión publicitaria en televisión abierta, misma que en el 2005 correspondió a 58\% (22 mil 150 millones de pesos) de la inversión publicitaria en medios masivos (AC Nelsen, 2006. Disponible en http://www.amap.com.mx/archivosdownload/segundo_estudio.swf). 
Sin más preámbulos vayamos al análisis comparativo entre los datos de 1999 (tabla 1) y 2003 (tabla 2) que nos arroja el SCIAN.28

\section{Producción bruta total}

En primer lugar, debemos destacar que en un lapso de cuatro años, entre 1999 y 2003, la producción bruta total de las industrias audiovisuales casi se duplicó, al generar 20 mil millones de pesos adicionales. Este incremento representó 0.57 y $0.67 \%$ del producto interno bruto (PIB) del país de 1999 y 2003,29 respectivamente, véanse tablas 1,2 y gráfica 1 . No obstante, ese crecimiento económico debemos observarlo de forma diferenciada para advertir que no todas las ramas y subramas del sector audiovisual crecieron; incluso advertimos una contracción importante en la subrama de la "producción de películas cinematográficas y videos" (512111), al reducir su producción en 35 millones de pesos. En ese sentido y correlativamente, la rama de "servicios de post-producción y otros servicios para la industria fílmica y del video" decreció 28.9 millones de pesos.

28 La selección de los rubros corresponden al sector de información en medios masivos (código 51). Se seleccionaron sólo aquellos subsectores, ramas y subramas que tienen que ver con las industrias audiovisuales; no se contempló a la industria musical, de la radio ni otras de telecomunicaciones que no tienen que ver con la televisión restringida. Esto con la idea de focalizar la observación en las industrias del cine, la televisión y el video, mismas que tienen las especificidades técnicas de audiovisuales.

Una primera versión del SCIAN se publicó en 1997. Sin embargo, paralelamente a su implementación fue revisándose y contrastándose el sistema con la idea de afinarlo a partir de su uso y de las sugerencias de especialistas en las distintas unidades económicas. En 2002 se publicó la nueva versión del SCIAN, siendo el sistema que estaba vigente al momento de realizar esta investigación. Es importante señalar que, por lo anterior, difieren algunos números de códigos entre los resultados presentados en 1999 y 2003. Sin embargo, las etiquetas o nombres son los mismos.

29 En esos años el PIB del país fue de 4,594,724,000,000 pesos y de $6,891,433,000,000$ pesos, respectivamente (www.banxico.org.mx). 


\section{TABLA 1}

INDICADORES ECONÓMICOS MÁS REPRESENTATIVOS DE LAS INDUSTRIAS AUDIOVISUALES MEXICANAS REPORTADOS POR EL SCIAN EN 1999

\begin{tabular}{|c|c|c|c|c|}
\hline $\begin{array}{l}\text { Código } \\
\text { SCIAN }\end{array}$ & Título & $\begin{array}{l}\text { Unidades } \\
\text { económicas }\end{array}$ & $\begin{array}{l}\text { Personal } \\
\text { ocupado }\end{array}$ & $\begin{array}{c}\text { Producción bruta } \\
\text { total } \\
\text { (en miles de pesos }\end{array}$ \\
\hline
\end{tabular}

\begin{tabular}{|c|c|c|c|c|}
\hline Subsector & Industria fílmica & 1,226 & 16,684 & $11,091,280$ \\
\hline 5121 & y del video. & & & \\
\hline Rama & Producción & & & \\
\hline \multirow[t]{6}{*}{51211} & de películas & & & \\
\hline & cinematográfica, & & & \\
\hline & $\begin{array}{l}\text { videos, } \\
\text { programas para }\end{array}$ & 407 & 7,668 & $6,662,092$ \\
\hline & la televisión y & & & \\
\hline & otros materiales & & & \\
\hline & audiovisuales. & & & \\
\hline Subrama & Producción & & & \\
\hline \multirow[t]{3}{*}{512111} & de películas & 87 & 407 & 191,806 \\
\hline & cinematográficas & & & \\
\hline & y videos. & & & \\
\hline Subrama & Producción de & & & \\
\hline \multirow[t]{2}{*}{512112} & programas para & 50 & 5,724 & $4,948,995$ \\
\hline & la televisión. & & & \\
\hline Subrama & Producción & & & \\
\hline \multirow[t]{4}{*}{512113} & de videoclips, & & & \\
\hline & comerciales y & 270 & 1,537 & $1,521,291$ \\
\hline & otros materiales & & & \\
\hline & audiovisuales. & & & \\
\hline Rama & Distribución & & & \\
\hline \multirow[t]{5}{*}{51212} & de películas & & & \\
\hline & cinematográficas, & 24 & 274 & 984,141 \\
\hline & videos y otros & & & \\
\hline & materiales & & & \\
\hline & audiovisuales. & & & \\
\hline
\end{tabular}




\begin{tabular}{|c|c|c|c|c|}
\hline $\begin{array}{l}\text { Código } \\
\text { SCIAN }\end{array}$ & Título & $\begin{array}{l}\text { Unidades } \\
\text { económicas }\end{array}$ & $\begin{array}{l}\text { Personal } \\
\text { ocupado }\end{array}$ & $\begin{array}{c}\text { Producción bruta } \\
\text { total } \\
\text { (en miles de pesos) }\end{array}$ \\
\hline \multirow{5}{*}{$\begin{array}{l}\text { Rama } \\
51213\end{array}$} & Exhibición & & & \\
\hline & de películas & & & \\
\hline & $\begin{array}{l}\text { cinematográficas, } \\
\text { videos y otros }\end{array}$ & 767 & 8,180 & $3,236,296$ \\
\hline & materiales & & & \\
\hline & audiovisuales. & & & \\
\hline \multirow{6}{*}{$\begin{array}{l}\text { Rama } \\
51219\end{array}$} & Servicios de & & & \\
\hline & posproducción & & & \\
\hline & y otros servicios & 28 & 562 & 208,751 \\
\hline & para la industria & & & \\
\hline & fílmica y del & & & \\
\hline & video. & & & \\
\hline \multirow{6}{*}{$\begin{array}{l}\text { Subrama } \\
51312\end{array}$} & Transmisión & & & \\
\hline & y repetición & & & \\
\hline & de programas & 519 & 10,845 & $9,814,282$ \\
\hline & de televisión, & & & \\
\hline & excepto a través & & & \\
\hline & de internet. & & & \\
\hline \multirow{5}{*}{$\begin{array}{l}\text { Rama } \\
5132\end{array}$} & Producción y & & & \\
\hline & distribución por & & & \\
\hline & suscripción de & 671 & 7,633 & $3,306,637$ \\
\hline & programas de & & & \\
\hline & televisión. & & & \\
\hline \multirow{5}{*}{$\begin{array}{l}\text { Rama } \\
5132\end{array}$} & Producción y & & & \\
\hline & distribución por & & & \\
\hline & suscripción de & 671 & 7,633 & $3,306,637$ \\
\hline & programas de & & & \\
\hline & televisión. & & & \\
\hline \multirow{5}{*}{$\begin{array}{l}\text { Subrama } \\
51321\end{array}$} & Producción & & & \\
\hline & de programas & & & \\
\hline & de televisión & 11 & 49 & 2,349 \\
\hline & distribuidos por & & & \\
\hline & suscripción. & & & \\
\hline
\end{tabular}




\begin{tabular}{llccc}
$\begin{array}{r}\text { Código } \\
\text { SCIAN }\end{array}$ & \multicolumn{1}{c}{ Título } & $\begin{array}{c}\text { Unidades } \\
\text { económicas }\end{array}$ & $\begin{array}{c}\text { Personal } \\
\text { ocupado }\end{array}$ & $\begin{array}{c}\text { Producción bruta } \\
\text { total } \\
\text { (en miles de pesos) }\end{array}$ \\
\hline $\begin{array}{l}\text { Subrama } \\
51322\end{array}$ & $\begin{array}{l}\text { Distribución por } \\
\text { suscripción de } \\
\text { programas de } \\
\text { televisión. }\end{array}$ & & 7,584 & $3,304,288$ \\
& 660 & & \\
TOTAL & & 2,416 & 33,162 & $24,212,199$ \\
\hline
\end{tabular}

Fuente: elaboración propia con datos del Sistema de Clasificación Industrial de América del Norte (SCIAN) 1997. Disponible en www.inegi.gob.mx.

\section{TABLA 2}

INDICADORES ECONÓMICOS MÁS REPRESENTATIVOS DE LAS INDUSTRIAS AUDIOVISUALES MEXICANAS REPORTADOS POR EL SCIAN EN 2003

\begin{tabular}{|c|c|c|c|c|}
\hline $\begin{array}{l}\text { Código } \\
\text { SCIAN }\end{array}$ & Título & $\begin{array}{l}\text { Unidades } \\
\text { económicas }\end{array}$ & $\begin{array}{l}\text { Personal } \\
\text { ocupado }\end{array}$ & $\begin{array}{c}\text { Producción bruta } \\
\text { total } \\
\text { (en miles de pesos) }\end{array}$ \\
\hline \multirow{9}{*}{$\begin{array}{l}\text { Subsector } \\
5121 \\
\text { Rama } \\
51211\end{array}$} & Industria fílmica & \multirow[t]{5}{*}{942} & \multirow[t]{5}{*}{6,284} & \multirow[t]{3}{*}{$26,286,480$} \\
\hline & y del video. & & & \\
\hline & Producción & & & \\
\hline & de películas & & & \multirow[t]{6}{*}{$18,875,676$} \\
\hline & cinematográficas, & & & \\
\hline & videos, programas & \multirow[t]{4}{*}{362} & \multirow[t]{4}{*}{2,563} & \\
\hline & para la televisión & & & \\
\hline & y otros materiales & & & \\
\hline & audiovisuales. & & & \\
\hline Subrama & Producción & & & \\
\hline \multirow[t]{3}{*}{512111} & de películas & \multirow[t]{4}{*}{41} & \multirow[t]{3}{*}{$\mathrm{S} / \mathrm{D}$} & \multirow[t]{3}{*}{156,047} \\
\hline & cinematográficas & & & \\
\hline & y videos. & & & \\
\hline Subrama & Producción de & & & \\
\hline 512112 & programas para la & 44 & $\mathrm{~S} / \mathrm{D}$ & $16,831,501$ \\
\hline
\end{tabular}




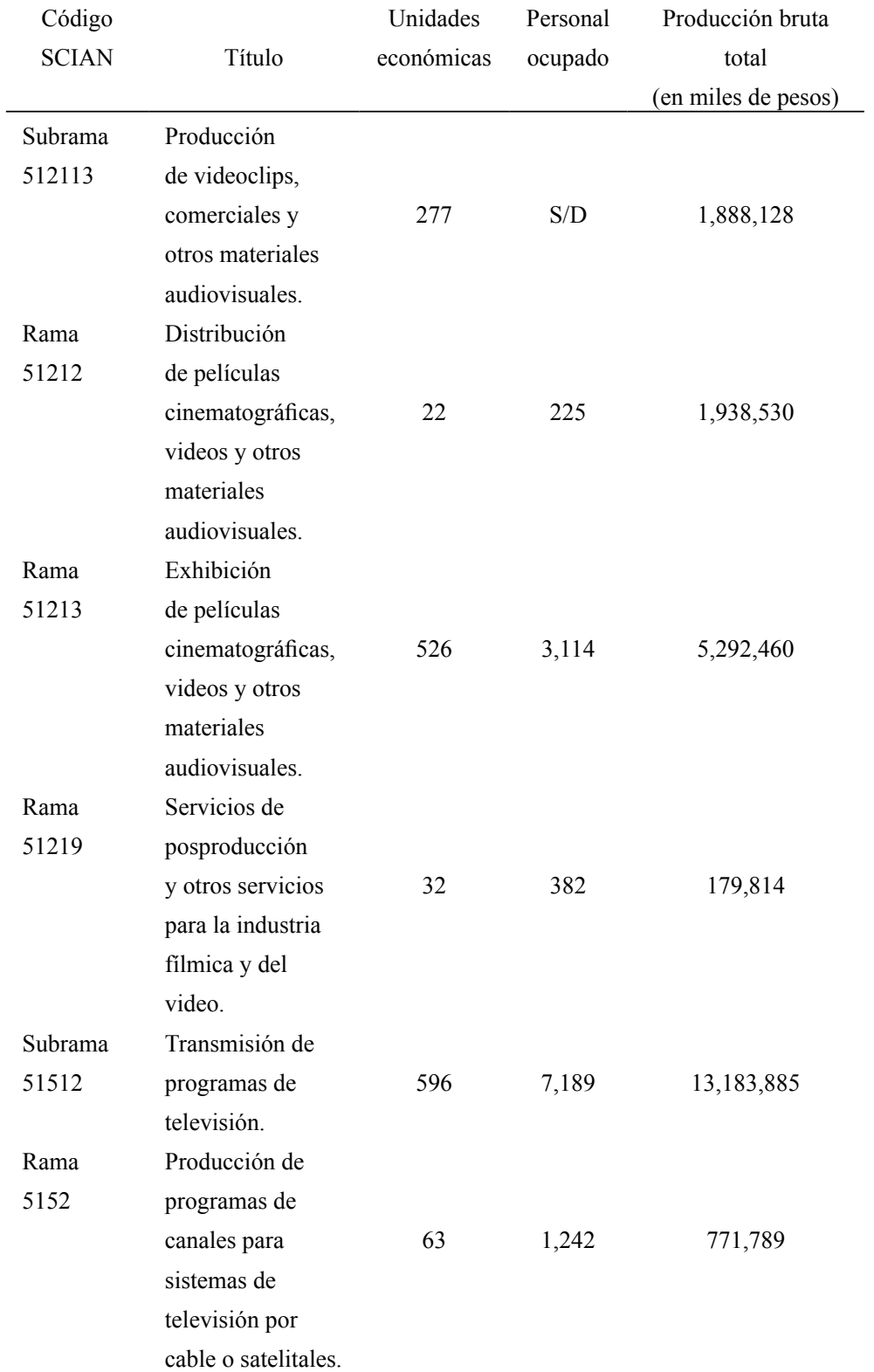




\begin{tabular}{llccc}
\multicolumn{1}{c}{$\begin{array}{c}\text { Código } \\
\text { SCIAN }\end{array}$} & \multicolumn{1}{c}{ Título } & $\begin{array}{c}\text { Unidades } \\
\text { económicas }\end{array}$ & $\begin{array}{c}\text { Personal } \\
\text { ocupado }\end{array}$ & $\begin{array}{c}\text { Producción bruta } \\
\text { total } \\
\text { (en miles de pesos) }\end{array}$ \\
\hline $\begin{array}{l}\text { Rama } \\
5175\end{array}$ & $\begin{array}{l}\text { Distribución } \\
\text { por suscripción } \\
\text { de programas } \\
\text { de televisión } \\
\text { (excepto a través }\end{array}$ & 1344 & 7,351 & $6,766,191$ \\
& de internet). & & & \\
& & & & \\
TOTAL & 2,882 & 20,824 & $47,008,345$ \\
\hline
\end{tabular}

Fuente: elaboración propia con datos del Sistema de Clasificación Industrial de América del Norte (SCIAN) 2002. Disponible en www.inegi.gob.mx.

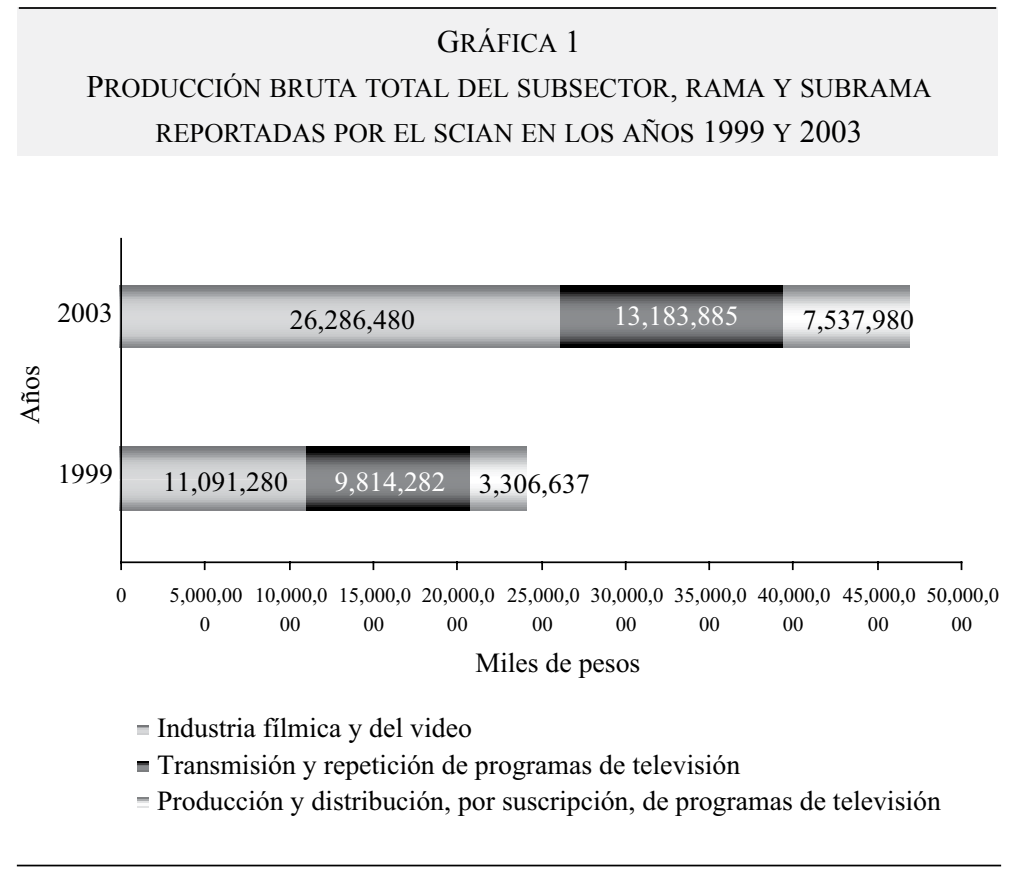

Fuente: elaboración propia con datos del SCIAN 1999 y 2003. Disponible en www.inegi.gob.mx. 
Esta tendencia confirma lo que diversas investigaciones han demostrado con respecto a otro tipo de indicadores -principalmente los relacionados con el número de producciones realizadas en esos años-, en el sentido de que la subrama audiovisual de la producción cinematográfica atraviesa por la peor crisis de su historia (Sánchez Ruiz, 2001; Gómez, 2005; Ugalde, 2006; Hinojosa, 2007).

En contraste, en 1999 y 2003 las ramas de "distribución y exhibición de películas cinematográficas, videos y otros materiales audiovisuales" crecieron en 100 y 80\%, respectivamente. En este sentido, se observa un claro desfase entre la rama de la producción y las ramas de la distribución y la exhibición de la industria cinematográfica en el país, al ser los productos estadounidenses los que más se distribuyen y exhiben en los circuitos cinematográficos, del video y de la televisión en México (Sánchez Ruiz, 2005; Ugalde, 2006:50-59). Además, cabe señalar que en las dos ramas beneficiadas se advierte una participación importante de capitales estadounidenses (Gómez, 2005), sobre todo en la rama de distribución, la misma que, de acuerdo con Nicholas Garnham, debemos considerarla como la clave del circuito audiovisual (Garnham, 1990).

Ahora bien, a partir de la observación del crecimiento de otros rubros encontramos que la subrama de la "producción de programas de televisión" (512113) es la palanca del crecimiento de la industria audiovisual en su conjunto, al incrementar su producción bruta total en $280 \%$, es decir, en 11.8 mil millones de pesos. Esta situación nos ofrece un indicador más para observar la concentración: la subrama de la televisión está dominada principalmente por las dos cadenas de ámbito nacional, Televisa y TV Azteca. Por ejemplo, en esos mismos años dichas empresas reportaban a la bolsa de valores de Nueva York que sus ingresos totales fueron, para la primera, de 2065 millones y de 2570 millones de dólares, respectivamente; 30 mientras que para la segunda fueron de 487 millones y 709

30 Estamos hablando de alrededor de 21 mil millones y de 28 mil millones de pesos, respectivamente. Asimismo, es importante señalar que de esos ingresos totales reportados por Televisa, 70\% tienen que ver con las industrias audiovisuales. Para mayor información véanse Informe 20 F 2000, 2002 e Informe Anual 2005 de Televisa. 
millones de dólares, ${ }^{31}$ respectivamente (Gómez, 2006). En otras palabras, estos datos permiten confirmar que la concentración no sólo se debe pensar en el rubro de la televisión abierta, sino de las industrias audiovisuales en su conjunto. Además, es importante subrayar que la empresa Televisa participa en casi todos los subsectores, ramas y subramas como uno de los actores más dominantes (sólo en la rama de la exhibición cinematográfica no participa el Grupo Televisa).

Finalmente, en este subapartado destacamos los resultados de las ramas que atañen a la televisión restringida, en la cual se confirma la tendencia observada de encontrarse en plena expansión (Gómez, 2007). La rama de "distribución por suscripción de programas de televisión” (5175) incrementó su producción bruta en 100\% (3.3 mil millones de pesos), mientras que la rama "producción por suscripción de programas de canales para sistemas de televisión por cable o satelitales" (5152) experimentó un crecimiento fuera de lo común, al pasar de los ridículos 2300000 pesos, a 771 millones de pesos. No obstante, cabe señalar que esta rama está subutilizada por la expansión y potencialidad de la televisión restringida, debido a la multiplicidad de sus señales y espacios para distribuir producción audiovisual nacional.

\section{Personal ocupado}

El rubro que tiene que ver con el personal que emplean las industrias audiovisuales nos permite advertir una de las principales características de las políticas neoliberales: priorizar la contratación temporal (free lance) sobre las plazas fijas. Con esto, las empresas buscan obtener los menores costos de operación - evadir pagos de seguridad social, pensiones, aguinaldos y prestaciones en general- para poder manejar de forma flexible sus recursos, por ejemplo, la posibilidad de recortes de personal a discreción. De ahí que se observe un recorte importante de personal entre los años que presenta la investigación. En total, estamos hablando de un recorte de 12338 plazas de trabajo. Lo anterior nos índica una

31 Para el caso de TV Azteca, son 5 mil y 7700 millones de pesos. 90\% de esos ingresos totales correspondieron al negocio de la televisión (Informe 20F, TV Azteca 2000 e Informe Anual, TV Azteca, 2005). 
clara vulnerabilidad de las condiciones laborales de los trabajadores relacionados con la producción audiovisual.

Otro punto que debemos anotar sobre las particularidades de los trabajadores del sector audiovisual es que tienen una movilidad importante entre los distintos subsectores, es decir, el periodista televisivo puede pasar a ser periodista radiofónico o el guionista radiofónico a ser guionista televisivo o cinematográfico, por lo que la baja en el rubro de personal ocupado es aún más alarmante. Esta situación contrasta claramente con el índice de crecimiento de la producción bruta total y con los objetivos gubernamentales de crear más empleos y de calidad.

\section{Unidades económicas}

Finalmente, en el índice que tiene que ver con el número de empresas que participan en las industrias audiovisuales también se advierte una clara baja en varios rubros claves, sobre todo en aquellos relacionados con la producción (véanse códigos 512111 y 512112) y la distribución (51212). Por otro lado, creció el número de empresas de "distribución por suscripción de programas de televisión" de forma importante; de hecho esta rama es la que impulsó el crecimiento de las empresas del sector audiovisual. Es justamente en esa rama donde podemos identificar una naciente competencia a nivel nacional entre las distintas plataformas de televisión restringida. No obstante, desde 2004 existe una posición monopólica en una de las plataformas de distribución (Sky). Sin embargo, después del Acuerdo de Convergencia de 2006, el cual permite la entrada de Teléfonos de México (Telmex) como distribuidor de contenidos audiovisuales, coloca a estas dos empresas de forma ventajosa por su extendida cobertura nacional, en detrimento de las compañías de cable y de súper alta frecuencia, pues ninguna de estas posee el alcance, la infraestructura y la liquidez financiera que sí tienen Sky (Grupo Televisa) y Telmex.

Otro factor que debemos señalar se refiere al tipo de empresas que están incrementándose en número; son las que tienen que ver con empleos no relacionados con la producción cultural, es decir, no se incrementa el número de empleos relacionados con la producción, sino sólo los que tienen que ver con la distribución y transmisión. Esta situación resulta alarmante en términos del impulso hacia la generación de 
empleos relacionados con la producción audiovisual y con la creación cultural y creativa.

\section{CONSIDERACIONES FINALES}

A partir de los datos aportados se puede profundizar en las particularidades de la concentración de las industrias audiovisuales en México. Esta industria se encuentra encapsulada en subsectores y ramas que tienen que ver con la producción y la transmisión de programas de televisión. Todo ello resulta en una situación contradictoria, pues si bien los resultados económicos nos muestran un crecimiento de casi $100 \%$ en el lapso de años presentado, también advertimos que las fuentes de empleo fijos se redujeron y el crecimiento de empresas del audiovisual se estancó. En contraparte, en esos mismos años Televisa y TV Azteca incrementaron sus ingresos totales de forma constante. En el caso de la primera, opera en todos los subsectores y ramas de la industria audiovisual; incluso conquistó una posición monopólica en el rubro de la televisión restringida vía satélite, al conseguir que el competidor de Sky, DIRECTV, se retirara del mercado nacional.

Por lo tanto, advertimos que si bien existe crecimiento económico, esto no refleja un desarrollo económico generalizado pues sólo algunos subsectores y ramas son los que han crecido, principalmente los que tienen que ver con las empresas dominantes del audiovisual. Es decir, el crecimiento se circunscribe en los dos conglomerados cuyo oficio base es la producción y transmisión televisiva, así como en las compañías de distribución de televisión restringida -cableras y Sky- y en las distribuidoras y exhibidores cinematográficos.

Así, me atrevo a sugerir que la estructura actual de las industrias audiovisuales, en México, no permite el desarrollo ni la incursión de nuevos actores. La concentración de los jugadores dominantes produce un efecto tapón: sólo a estos grandes jugadores se les permite un crecimiento constante y una posición privilegiada para competir a nivel internacional. A esta fórmula se le ha denominado "concentrar para competir" (Sinclair, 1996; Sánchez Ruiz, 2005).

Ante los datos aquí expuestos, considero que es imperioso impulsar políticas normativas que reorienten el desarrollo de las industrias au- 
diovisuales en México. Se advierten monopolios y prácticas monopólicas que supuestamente las administraciones Salinas-Zedillo-Fox buscaron erradicar. Lo anterior consiste en una de las principales banderas y presumibles bondades de la economía de mercado y de libre comercio, sobre todo en términos de competencia económica dirigida a conseguir un desarrollo económico. De tal suerte que dentro de la retórica de las políticas neoliberales no están cumpliéndose algunos de sus principales objetivos y presupuestos.

Sin embargo, podemos afirmar que mientras persista la ausencia de mecanismos para construir una gobernabilidad democrática participativa -sobre todo en el proceso de diseño, elaboración y evaluación de políticas públicas-, en el cual concursen los distintos agentes sociales involucrados en el sistema audiovisual mexicano, no estaremos en posibilidad de construir las condiciones necesarias, como país democrático, para trazar un paisaje audiovisual acorde con las demandas, las necesidades y la diversidad de la dinámica sociedad mexicana del siglo XXI.

Para finalizar, se proponen las siguientes líneas de acción que deben de abordar el diseño de políticas audiovisuales con carácter nacional, para poder conseguir objetivos orientados hacia el desarrollo económico, democrático y sociocultural:

- Cambiar el modelo de construcción, diseño y negociación de las políticas audiovisuales, controlado por las empresas privadas dominantes y las elites políticas afines a los conglomerados, para transformar los esquemas de gobernabilidad hacia una participación activa de la sociedad civil;

- Incorporar políticas normativas que financien y protejan a la producción nacional audiovisual -por ejemplo, impuestos directos a las ganancias de los concesionarios privados y a la taquilla, y repensar la figura de un Banco Nacional para la Industria Audiovisual-;

- Frenar y limitar el crecimiento horizontal, vertical y cruzado de los conglomerados del audiovisual;

- Armonizar el ingreso de capitales extranjeros en el subsector de la radiodifusión y telecomunicaciones hasta en $25 \%$;

- Promover una competencia económica que permita el ingreso y el desarrollo de las pequeñas y medianas empresas del sector audiovisual; 
- Armonizar el marco jurídico de la industria audiovisual a partir de un anclaje y justificación cultural y social sobre las determinantes tecnológicas y económicas;

- Incluir distintas formas emergentes de producción y difusión audiovisual (medios comunitarios, locales, ciudadanos, universitarios, etcétera) para promover la diversidad cultural, las identidades locales, la pluralidad política, la libertad de prensa y de expresión, la comunicación de proximidad y el desarrollo social;

- Impulsar los sistemas de radiodifusión públicos -por medio del acceso universal, financiamiento, digitalización, continuidad y autonomía organizativa- como contrapeso a la lógica de producción y operación comerciales; y

- Estructurar la oferta audiovisual de manera que las diversas clases sociales, etnias y grupos puedan reconocerse con sus diferencias, lograr una distribución más justa de los recursos simbólicos y acceder tanto a los consumos audiovisuales como a la creación y producción audiovisual (García Canclini, 2004).

\section{Bibliografía}

AGUILAR, Luis F. (2004) "Recepción y desarrollo de la disciplina de política pública en México. Un estudio introductorio”, Sociológica, UAM-A, núm. 54, México.

BUSTAMANTE, Enrique (coord.) (2003) Hacia un nuevo sistema mundial de comunicación. Las industrias culturales en la era digital, Barcelona: Gedisa.

CASAS PÉREZ, María de la Luz (2006) Políticas públicas de comunicación en América del Norte, Limusa-ITESM, México.

CROTEU, David y William Hoynes (2001) The business of media. Corporate media and the public interest, Pine Forge Press, Thousand Oaks, California.

Crovi, Delia (1997) "Inequidades del NAFTA/TLCAN: un análisis del sector audiovisual”, en Mastrini G. y C. Bolaño (eds.) Globalización y monopolios en la comunicación en América Latina. Hacia una Economía Política de la Comunicación, Buenos Aires: Biblos.

CRUSAFON, Carmina (2000) El espacio audiovisual europeo: análisis de la industria audiovisual y de las políticas europeas en la década 
de los noventa, tesis doctoral, Bellatera, Universidad Autónoma de Barcelona.

CuilenburG, J. Van y Dennis McQuail (2003) "Media policy paradigm shifts. Towards a new communication policy paradigm", $E \mathbf{E}$ ropean Journal of Communication, vol. 18 (2), London: Sage.

CURRAn, James y Jean Seaton (1997) Power with out of responsibility, London: Routledge.

EUROPEAN Audiovisual Observatory (2003) Statistyc Yearbook, vol. 3, Strasbourg, Conseil de l'Europe, 2003.

FORMAN, P. y R. Saint John (2000) "Creating convergence", Scientific American, EEUU, noviembre de 2000.

FRAU MEIGS, Divina (2003) “'Excepción cultural', políticas nacionales y mundialización: factores de democratización y de promoción de lo contemporáneo", Quaderns del CAC, núm. 14, Barcelona.

GALPERIN, Hernán (1999) "Cultural industries policy in regional trade agreements: the case of NAFTA, the European Union and MERCOSUR", Media, Culture \& Society, 21 (5).

GARCÍA CANCLINI, Néstor y Ernesto Piedras (2006) Industrias culturales y el desarrollo en México. Siglo XXI-FLACSO-SER, México.

- (2004) Diferentes, desiguales y desconectados. Mapas de la interculturalidad, Barcelona: Gedisa.

- y J.C. Moneta (coords.) (1999) Las industrias culturales en la integración latinoamericana, México: Grijalbo.

- (ed.) (1987) Politicas culturales en América Latina, México: Grijalbo.

GARNHAM, Nicholas (2005) "From cultural to creative industries. An analysis of the implications of the 'creative industries' approach to arts and media policy in the United Kingdom", International Journal of Cultural Policy, vol. 11, núm. 1.

- (1990) Capitalism and Communication, Londres: Sage.

GIFREU, Joseph (1986) El debate internacional de la comunicación, Barcelona: Ariel.

GOLDING, Peter y Graham Murdock (2000) "Culture, communications, and political economy", en Curran J. y M. Gurevitch, Mass media and society, Edward Arnold, tercera edición, Londres.

- (1998) "New technologies and old problems: evaluating and re- 
gulating media performance in the "information age", en Brants, Hermes y Van (eds.), The media in question. Popular cultures and public interests, Londres: Sage.

GÓMEZ, Rodrigo (2007) "Políticas de comunicación en México 19882006. El giro neoliberal", en Gómez, R. y A. Peimbert, Comunicación para el desarrollo en México. Libro colectivo AMIC 2006, México: ULA-AMIC.

- (2006) El impacto del Tratado de Libre Comercio de América del Norte en la industria audiovisual mexicana (1994-2002), tesis doctoral, Universidad Autónoma de Barcelona, Bellaterra.

- y Gabriel Sosa (2006) "Reforma de la legislació en radio, televisió i telecomunicacions a Méxic", Quaderns del CAC, Barcelona, Catalunya, núm. 25, mayo-agosto de 2006.

- (2005) "La industria cinematográfica mexicana. Estructura, desarrollo, políticas y tendencias 1992-2003”, Estudios sobre las culturas contemporáneas. Revista de investigación y análisis, Colima, México, segunda época, vol. X, núm. 22, diciembre de 2005.

- (2004) "TV Azteca y la industria audiovisual mexicana en tiempos de integración regional (TLCAN) y desregulación económica”, Comunicación y Sociedad, Guadalajara, núm. 1, nueva época.

HERSCOVICI, Alain (2005) "Economía de la comunicación, lógicas sociales y territorialidad", en Bolaño, César, Guillermo Mastrini y Francisco Sierra, Economía política, comunicación y conocimiento. Una perspectiva crítica latinoamericana, Argentina: La CrujíaJunta de Andalucía.

HESMONDHALGH, David (2002) Cultural industries, Londres: Sage. HINOJOSA, Lucila (2007) "El cine mexicano y el Tratado de Libre Comercio de América del Norte (TLCAN): un análisis multidimensional”, en Gómez, R. y A. Peimbert (2007) Comunicación para el desarrollo en México. Libro colectivo AMIC 2006, México: ULAAMIC, .

INSTITUTO NACIONAL de Estadística, Geografía e Informática (2002) Sistema de Clasificación Industrial de América del Norte (SCIAN) México, 2002, Aguascalientes: INEGI.

MATTELART, Armand (1998) La mundialización de la comunicación, Barcelona: Paidós. 
MCBRIDE, Sean (1987) Un solo mundo, voces múltiples. Comunicación e información en nuestro tiempo, México: Fondo de Cultura Económica.

MCQUAIL, Dennis (1998) La acción de los medios. Los medios de comunicación y el interés público, Amorrortu, Argentina: Buenos Aires.

MIÈGE, Bernarde (1989) "The logics at work in the new cultural industries", Media, Culture \& Society, vol. 9.

MosCO, Vincent (1996) The political economy of communication, Londres: Sage.

MURCIANO, Marcial (1992) Estructura y dinámica de la comunicación internacional, Barcelona: Bosch.

MURDOCK, Graham (1998) "Comentarios de base: las condiciones de la práctica cultural”, en Ferguson y Golding (eds.). Economía política y estudios culturales, Bosch, Barcelona.

QUALTER, Terence (1994) Publicidad y democracia en la sociedad de masas. Paidós, Barcelona.

PASQUALI, Antonio (1994) "Reinventar los sistemas públicos", Nueva Sociedad, núm. 140, noviembre-diciembre, Caracas.

PAUWELS, Caroline y J. Loisen (2003) "The WTO and the audiovisual sector. Economic free trade vs cultural horse trading?", European Journal of Communication, vol. 18, Londres: Sage.

PICARD, Robert (2002) The economics and financing of media companies, EUA: Fordham University Press.

Ramos, Nubia (2007) Políticas de comunicación para las culturas populares en México (1978-2006). El caso de la Dirección General de Culturas Populares, tesis de maestría, México: UNAM.

RICHERI, Guissepe (ed.) (1983) La televisión: entre servicio público y negocio, Barcelona: Gustavo Gili.

SÁNCHEZ Ruiz, Enrique (2005) "El audiovisual mexicano: ¿concentrar para compartir?", Global Media Journal en español, vol. 1, núm. 2, primavera de 2005. Disponible en http://gmje.mty.itesm.mx/articulos2/sanchezruiz_OT04.html.

- (2003) "El estado de los medios en Iberoamérica. Particularidades del mercado mexicano de la televisión”, Revista Telos, núm. 57.

- (2001) "Globalization, cultural industries, and free trade: the mexican audiovisual sector in the NAFTA Age", en Mosco, V. y D. Schi- 
ller (ed). Continental order? Integrating North America for cybercapitalism, EUA: Rowman \& Littlefield.

- (1991) Medios de difusión y sociedad. Notas críticas y metodológicas, México: Universidad de Guadalajara.

SIERRA, Francisco (2006) Políticas de comunicación y educación: crítica y desarrollo de la sociedad del conocimiento, Barcelona: Gedisa.

- (2002) Bases de la política audiovisual europea, Mergablum, Sevi$11 \mathrm{a}$.

SINCLAIR, Jhon (1996) "Mexico, Brazil and the latin world", en Sinclair, Jhon, E. Jaka y Stuart Cunningham (eds.), New patterns in global television. Peripheral vision, New York: Oxford University Press.

TELEVISA (2004) Informe Anual, 2003, México: Televisa.

- (2003) Informe Anual 2002, México: Televisa.

- (2001) Annual report 2000 form 20F, México: Televisa.

TORRENT, Ramón (2003) 'La 'excepción cultural' en la Organización Mundial del Comercio (OMC): la base de la política audiovisual en Cataluña", Quaderns del CAC, núm. 14, Barcelona.

TOUSSAINT, Florence (1998) Televisión sin fronteras, México: Siglo XXI.

TV AZTECA (2004) Informe Anual 2003, México: TV Azteca.

- (2003) Informe Anual 2002, México: TV Azteca.

- (2001) Annual report 2000 form 20F, México: TV Azteca.

UGALDE, V (2006) "Panorama de la producción cinematográfica nacional”, Estudios cinematográficos-UNAM, núm. 26.

\section{Enlaces consultados en internet}

www.banxico.org.mx

www.inegi.gob.mx

www.amap.com.mx 